\title{
Simulation of guard ring influence on the performance of ATLAS pixel detectors for inner layer replacement
}

\author{
M. Benoit, ${ }^{1,2}$ A. Lounis ${ }^{2}$ and N. Dinu ${ }^{2}$ \\ Laboratoire de l'accélérateur linéaire, \\ Orsay, France \\ E-mail: Benoit@lal.in2p3.fr
}

ABSTRACT: Electric field magnitude and depletion in the bulk of silicon pixel detectors, which influence its breakdown behaviour, was studied using finite-element method to solve the driftdiffusion equation coupled to Poisson's equation in a simplified two dimensional model of the ATLAS pixel sensor. Based on this model, the number of guard rings and dead edges width were modified to investigate their influence on the detector's depletion at the edge and on its internal electrical field distribution. Finally, the 3 level model was implemented into the simulation to study the behaviour of such detector under different level of irradiation.

KEYWORDS: Solid state detectors; Simulation methods and programs; Detector modelling and simulations II (electric fields, charge transport, multiplication and induction, pulse formation, electron emission, etc); Radiation damage to detector materials (solid state)

\footnotetext{
${ }^{1}$ Corresponding author.

${ }^{2}$ On behalf of ATLAS Planar Pixel Upgrade Group
} 


\section{Contents}

1 Introduction 1

2 Theory 2

3 Simulation goals $\quad 4$

4 Simulated model $\quad 5$

5 Results 6

$\begin{array}{lll}5.1 & \text { Current ATLAS planar pixel sensor design } & 6\end{array}$

$\begin{array}{ll}5.2 \text { Dead edge reduction } & 8\end{array}$

5.3 reducing the number of guard rings 8

6 Discussion $\quad 8$

$\begin{array}{lll}7 & \text { Conclusion } & 10\end{array}$

\section{Introduction}

The inner layer of ATLAS pixel detector will see a fluence of $5.4 \times 10^{14} n_{\mathrm{eq}} / \mathrm{cm}^{2}$ after a year of operation [1]. A replacement of the inner detector, unable to withstand that level of irradiation, will be necessary for the operation of the ATLAS detector at super-LHC regime. The new inner layer's pixel detector will be required to deliver sufficiently high signal after high level of irradiation thus its associated electronics will require to process low signal with very low threshold.It is also desired to reduce the thickness of the inner detector to reduce its influence on Bremsstrahlung photons produced by electrons crossing the inner layer and to reduce the amount of radiation damage taken by the detector. Finally, limited budget and resources makes cost versus efficiency a major factor to consider in the choice of the super-ATLAS inner detector.

Technology computer-assisted design (TCAD) uses our knowledge of partial differential equation that describe charge carrier motion and interaction with the crystal lattice in semiconductors, coupled to finite element method to simulate the physical parameter of the device. This method can be used to explore different design for a device before its production to optimize different parameters. We used this method to explore different possible designs for the super-ATLAS planar pixel design. The influence of many design parameters such as the number and spacing of guard rings and inactive edge width, on detector electrical characteristics, based on simulation, are presented. A simple model for radiation damage have also been implemented to simulate the effect of radiation damage. From our simulation results, we suggest guideline for the design of future pixel prototypes and suggest test structure to be built to verify and adjust our model. 


\section{Theory}

Charge carrier dynamics in semiconductors like Silicon has been shown to be well described by the drift diffusion equation (2.1), (2.2), coupled to the Poisson equation (2.3) [2].

$$
\begin{aligned}
\frac{d p}{d t} & =\nabla \cdot D_{h} \nabla p+\nabla \cdot\left(p \mu_{h} \vec{E}\right)+G_{h}-\tau_{h} \\
\frac{d n}{d t} & =\nabla \cdot D_{e} \nabla n-\nabla \cdot\left(n \mu_{e} \vec{E}\right)+G_{e}-\tau_{e} \\
-\nabla^{2} V & =\nabla \cdot \vec{E}=\frac{\rho}{\varepsilon} \\
J_{\text {disp }} & =\varepsilon \frac{\partial \vec{E}}{\partial t}
\end{aligned}
$$

$p$ and $n$ are respectively the density of holes and electrons in $\left[\frac{1}{\mathrm{~cm}^{3}}\right], D$ in $\left[\frac{\mathrm{cm}^{2}}{\mathrm{~s}}\right]$, their respective diffusion coefficient, $\mu$ the mobility in $\left[\mathrm{cm}^{2} / V / s\right] . G$ is the generation rate and $\tau$, the recombination rate, both in $\left[1 / \mathrm{cm}^{3} / \mathrm{s}\right]$. The $h$ and $e$ subscript respectively design holes and electrons. $\rho$ is the net charge density in $\left[C / \mathrm{cm}^{3}\right]$, where $\mathrm{C}$ are Coulomb. $\overrightarrow{J_{\text {disp }}}$ is the displacement current density in $\left[C / \mathrm{cm}^{3} / \mathrm{s}\right]$ which need to be considered in transient solution, and $\varepsilon$ is the material dielectric constant.

The model used here to represent the density of state at equilibrium for free carriers, $n$ and $p$, is Boltzmann statistics, which is valid when $k_{b} T$ is negligible in front of $E_{c}-E_{f}$, where $E_{f}$ is the Fermi energy level and $E_{c}$ is theconduction band energy, as it is the case for our simulation. $k_{b}$ is the Boltzmann constant and $\mathrm{T}$ the temperature.

Generation/Recombination terms are important to describe the behavior of silicon detectors. Generation is responsible for leakage current present in detectors under bias. Recombination is important to describe transient behavior of carriers the device after perturbation by a charged particle crossing the depleted bulk. Silicon being an indirect gap semiconductor, generation and recombination occurs mostly through the defect states that are present in the bandgap of Silicon. The model used in the simulation is Shockley-Read-Hall Generation-Recombination [2], which describe generation-recombination in indirect-bandgap semiconductors as Silicon. This model approximates that the transition of carriers in the bands occurs through a single trap level located at midgap, $E_{\text {trap }}$.

High resistivity Silicon used for detectors is not pure. The presence of Oxygen and other dopants affects its electrical properties. Dopant are also introduced during fabrication of the detectors. Defects are also introduced by high energy particles crossing the detector. In the super-LHC environnement, ATLAS inner tracker will be submitted to high level of radiation and the introduction of structural defects must be taken into account in the design of the detectors.

Our simulation include a modified Shockley-Read-Hall Generation-Recombination model that takes into account the presence of multiple trap level in the band gap. Generation-Recombination term for each trap is calculated and a global term $R_{\text {total }}$ is calculated following 2.5 .

$$
R_{\text {total }}=\sum_{\alpha=1}^{l} R_{D_{\alpha}}+\sum_{\beta=1}^{m} R_{A_{\beta}}
$$


$l$ and $m$ are the number of donor and acceptor traps, and $R_{D, A}$ the Generation-Recombination term for each donor and acceptor traps. Density of traps are taken into account through empirical parameters $\tau_{n}$ and $\tau_{p}$ used for each trap level.Finally, charge state of traps are taken into account in Poisson equation right term. The amount of ionized trap is determined using Boltzmann statistics.

Radiation damage introduce defects in the bulk of the silicon that modify its behavior. A model of defect energy state distribution exist to try to reproduce the behavior of irradiated detector. The model allow one to reproduce the depletion potential [3], the douple peak in the electric field after irradiation and space charge sign inversion (SCSI) for n-bulk detectors [4-6].

The presence of high bias voltage on the detector result in high electrical field inside the bulk. When the electrical field is sufficiently high, breakdown can occur due to acceleration of free carriers in the electric field. Accelerated electrons ionize surrounding atoms creating an avalanche.The Selberherr's Impact Ionization Model [7] has been used to account for impact ionization in our simulations.

To solve our set of differential equation we need to restric ourself to a solution in a bounded domain, the detector. We must choose boundary conditions that are mathematically possible and reflects the properties of the system we want to simulate. Three types of boundaries were used during our simulation, representing the oxide-Silicon interface, the electrode interface, and the periodicity boundary. In addition we need a model for the cutting edge of the detector. The boundary between Si Dioxide and Silicon is a semiconductor/insulator boundary characterized by the presence of an accumulated charge layer at the interface. The boudary condition applied to these surface for Poisson equation is the Neumann boudary condition that takes into account the charge layer present at the surface. Also, electrons and holes concentrations is set to 0 on this boundary and the current is not allowed to flow through it.

Metal-semiconductor surface is the boundary between the silicon bulk and the metallic electrodes. This is a ohmic contact and current is allowed to flow through it. Electric potential $\Phi$ is fixed at one electrode to correspond to the bias voltage applied to the detector. The concentration of carriers is determined by Boltzmann statistics, knowing the potential applied at the electrodes. The effect of contact workfunction is considered negligible as high doping region are located above the electrodes.

Guard ring structure is metal semi-conductor interface where the metallic electrode is left floating. To represent this case, we must impose a fixed potential and a null current flow on this contact. The potential taken by the floating contacts are then found starting from an initial guess, using an iterative method.

To reduce the size of the problem to be solved, we can use periodicity boundary conditions using geometric properties of the detector. In our simulation, we will be interested to the solution on the sides of the detector. Knowing the solution will become periodic in the X-Y plane when approaching the center of the device, we can cut our model at a distance of the edge large enough to consider the solution will become periodic at this point. We then impose the periodicity condition at the surface for electron and hole concentrations and for electric potential. 


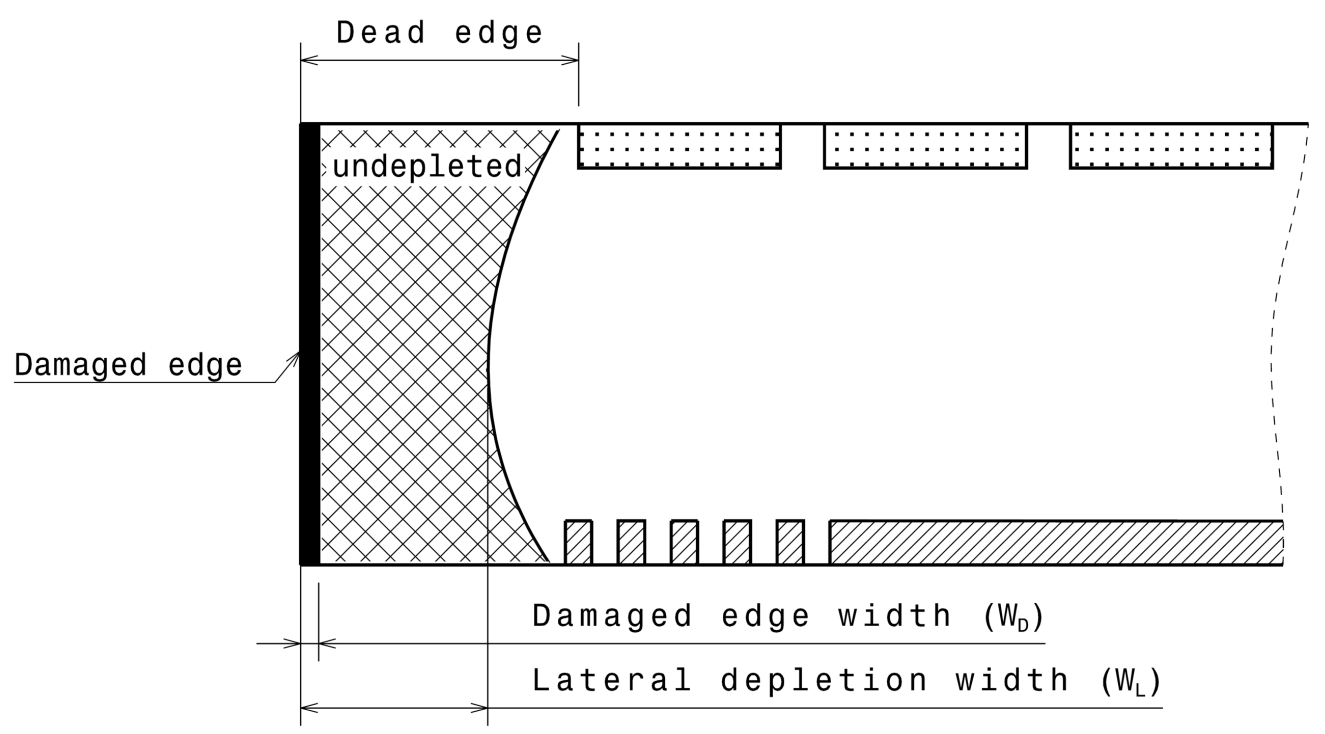

Figure 1. Lateral depletion at the edge of the detector.

\section{Simulation goals}

This section details the different characteristics of the detector we want to simulate and the information that we can extract from the result to obtain information on the possible behavior of real detectors.

The full depletion voltage is an important parameter of semiconductor detectors. As the bias potential on the detector electrode increase, the amount of free carriers in the bulk of the detector is reduced, up to the point where all the carriers are gone. The potential where this condition is reached is the depletion potential. In pixel detectors, the signal generated by carriers deposited by charged particles crossing the detector is proportional to the length of the track in the depleted bulk of the detector. Under-depleted or undepleted pixels will see less or no charge when crossed by a charged particle. Knowing the full depletion voltage of a detector also allow us to know the voltage at which it needs to be operated. In addition, radiation damage caused by exposition to high particle fluence will modifiy the depletion potential by modifying the effective dopant concentration in the bulk [8].

Lateral non-depletion width $W_{L}$ is another related parameter that tells us the distance from a detector's edge where the Silicon is undepleted. This has proven to be important to predict the occurance of lateral breakdown between pixel and guard ring structure. Edges of detectors are characterized by a high concentration of defects. The cutting of the detector, its oxydation and the diffusion of impurities and the propagation of damage in the lattice on a certain depth $W_{d}$, that is dependent of the dicing method used to cut the detector from it originating wafer, influence the edge behavior. If an electric field reach this region $\left(W_{L}<W_{d}\right)$, the high density of defects will increase the Generation term of the drift-diffusion equation, generating high leakage current and eventually breakdown [9]. In our simulation, we pay close attention to the determination of $W_{L}$ to ensure it is larger than the $W_{d}$ associated to the detector's dicing method.

The Electric field shape inside the bulk of the detector is an important parameter to determine 
its charge-sharing behavior and its typical pulse shape. The free carriers move in the electric field and its magnitude influence the speed at which the charge is collected. Most importantly, as mentionned before, electric field magnitude tell us about the probability of breakdown due to avalanche formation. Breakdown electric field in Silicon is in average close to $\left|E_{\text {break }}\right|=3 \times 10^{5} \mathrm{~V} / \mathrm{cm}$. To be possible as a detector configuration, our model should not present a field magnitude over this limit.

\section{Simulated model}

Our goal in this simulation is not to reproduce in details the behavior of the ATLAS pixel detector, but extract tendencies and offer guidelines for the design of future detectors. Hence, we simplified our model by using a 2D simulation geometry represented in figure 1 . This model stays valid as we considered that detector to be half-infinite and symetric in the YZ plane. We have built a parametric model of detector that can be used to explore its behavior while changing the different characteristics of the model. By reducing the size of our model comparatively to the real detector geometry, we obtain a problem that is easily solved in terms of minutes, which allow us to explore a large range of parameters in a reasonable simulation time.

The doping concentration used for our model is taken from ATLAS pixel Technical Design Report [1]. The bulk is high resistivity n-type Silicon $(2 k \Omega \mathrm{cm})$. Pixel doping is n-type and are insulated from each other by p-spray. Guard ring and high-voltage electrode doping are p-type. We chose to use simplified representation of doping profiles as real doping profiles of the detector are not well known. Doped region are 1 micron deep and decay exponentially over 1 microns outside the doped zone. This is coherent with monte-carlo simulation of ion implantation in silicon. This simplifies the problem, hence reducing computing time needed. The goal of our simulation is not to reproduce exactly the behavior of detectors so this level of accuracy is sufficient for our simulation needs.

The aim of the guard ring structure present next to the high-voltage electrode is to ensure a smooth transition from high voltage to ground while approaching the outer edge of the device.In an ATLAS pixel detector, the number of guard ring is fixed to 16, with a width of 10 microns and a distance between them varying from 15 to 8 microns. A metal overhang of various width is present over the oxide on the high voltage side of the guard ring and serves to control the oxide charge distribution and the electric field in the oxide.

The dicing of pixel detectors from their originating wafer create structural damage that affects the properties of the edge. A dead edge width must be included in the design, as shown in figure 1, to exclude this zone from the sensible part of the detector. This dead edge adds to inactive part of the detector and must be kept as small as possible. A special attention must be taken to modelize the dead edge of a silicon detector. Dicing mechanism is introducing structural damage in the Silicon crystal lattice close to the cutting region. This induce a process of amorphisation of silicon. Amorphous Silicon is a complex material because no short or long distance order in the crystal lattice. A way to modelize amorphous $\mathrm{Si}$ is to introduce a high number of defects in the bandgap of Silicon. As the crystal lattice of the Silicon is highly perturbated in the cutting edge region, trap states are created by the defaults in the crystal lattice that are introduced. To represent such a distribution of defects in the bandgap, we use a continuous density of states distribution to describe 


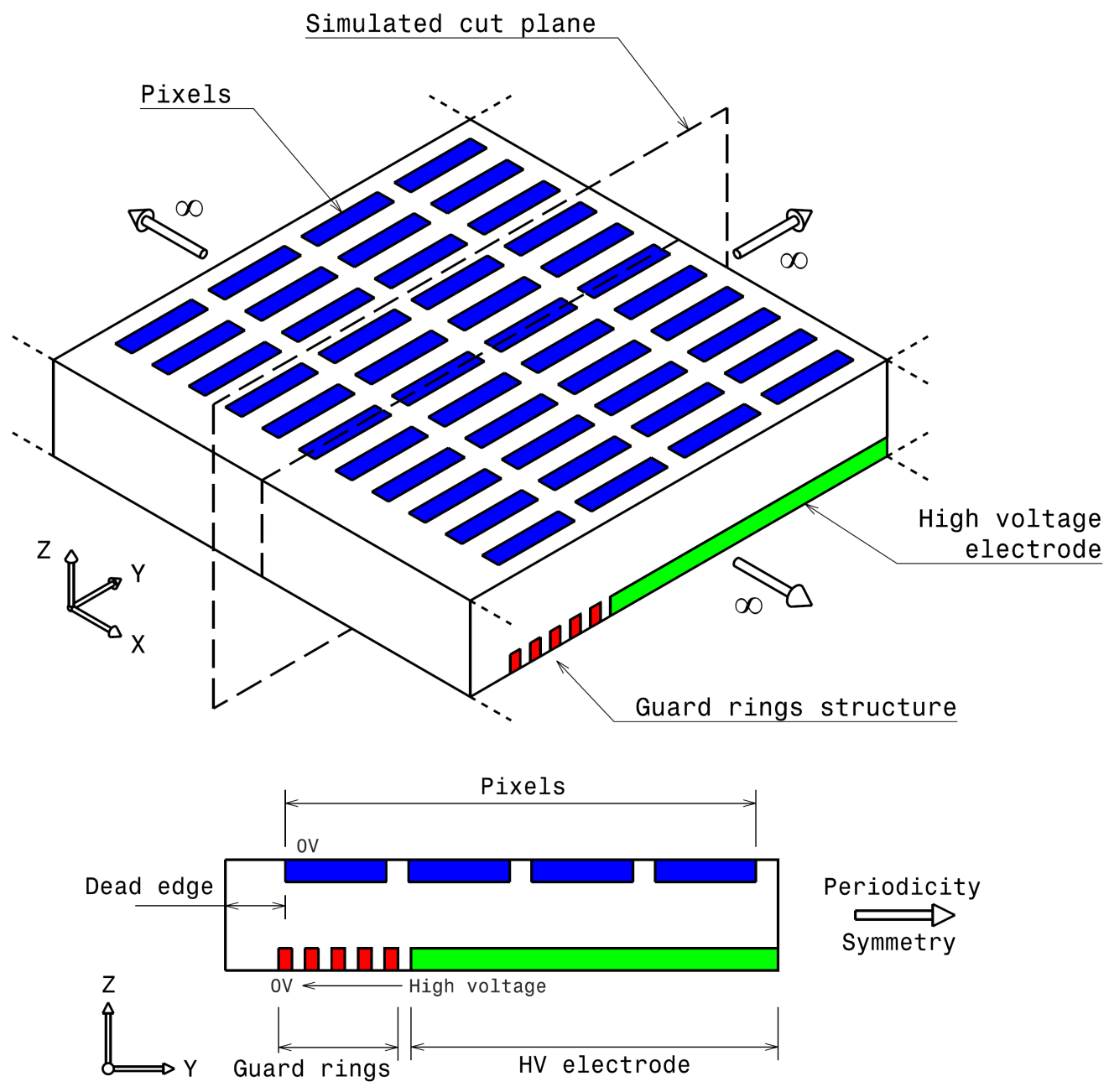

Figure 2. Simulation geometry, 3D view of the idealized detector (left) and $\mathrm{Y}-\mathrm{Z}$ simulated plane (right).

the bandgap defect structure. This distribution has the effect of making the edge conductive. The model used in these simulation is the one proposed by E. Noschis and al. [10].

\section{Results}

For this simulation study, we decided to concentrate on exploring the feasability of reducing the inactive surface of planar pixel detectors by reducing the number of guard rings and the width of the dead edge left on the side for protection. We will presnt simulation of the current design and explore how the reduction of the dead edge width, of the number of guard rings or the thinning of the detector can help reducing the inactive area of planar pixel sensors.

\subsection{Current ATLAS planar pixel sensor design}

Simulation of the current ATLAS planar pixel sensor design was performed up to 500V for unirradiated and irradiated detector. The 3-level model has been used to simulate radiation damage. 

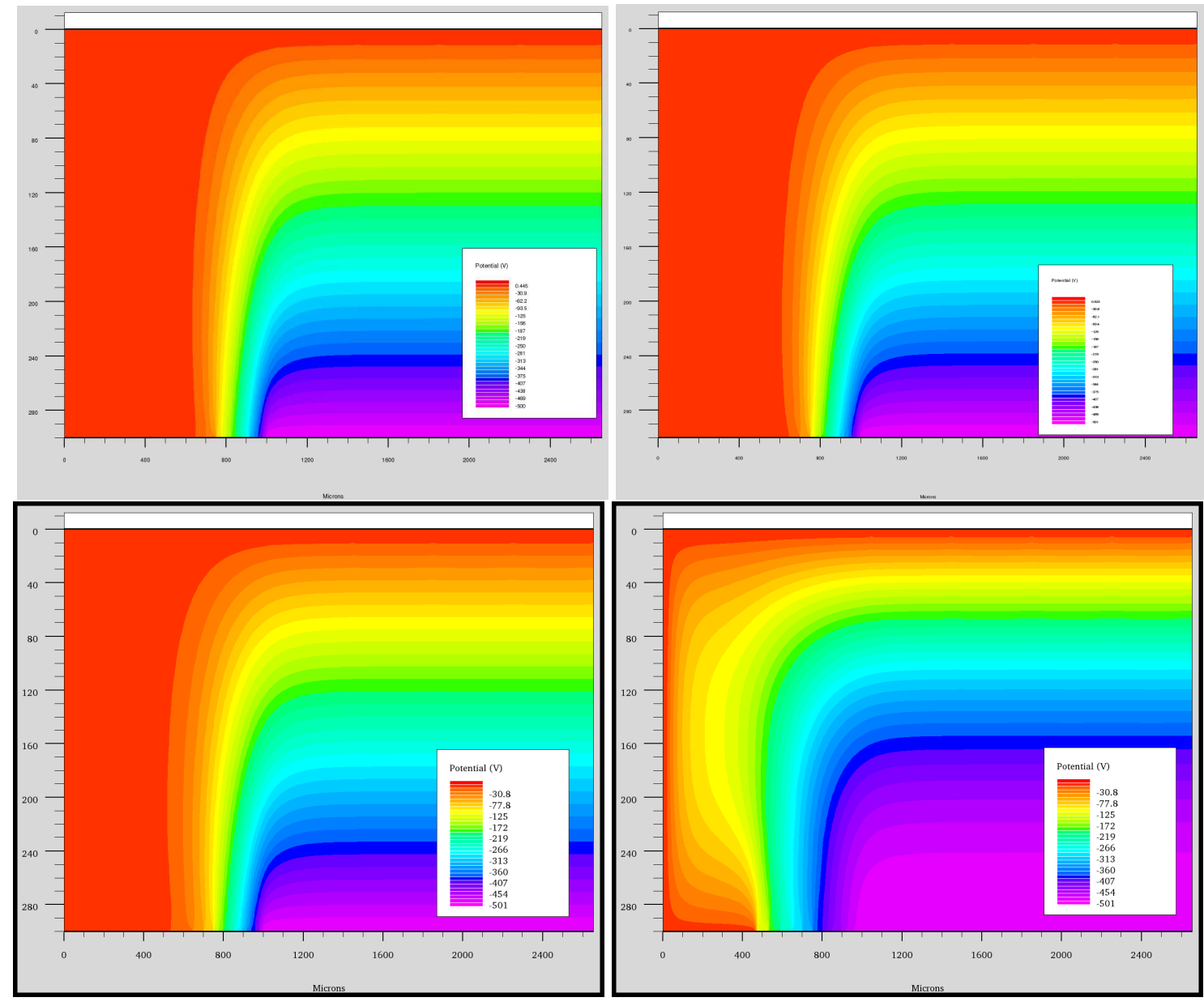

Figure 3. Simulated Potential profile for ATLAS pixel detector, unirradiated (upper left), $1 e 12 n_{\mathrm{eq}} \mathrm{cm}^{-2}$ (upper right), $1 e 13 n_{\mathrm{eq}} \mathrm{cm}^{-2}$ (bottom left), $1 e 14 n_{\mathrm{eq}} \mathrm{cm}^{-2}$ (bottom right), Minimum=0V maximum=-500V.

Figure 3 shows the simulated potential for the Actual ATLAS pixel design. Four pixel are included along with the guard rings (to the left of the figures). As fluence seen by the detector increase, the bulk type invert and depletion occurs from pixel toward the backplane.

Figure 4 shows the simulated potential distribution on the guard ring structure after exposition different fluences. Guard ring takes potential as the depletion region of te detector reach them. After SCSI, depletion occurs from the pixel side and may never completely reach the guard ring side of the detector. This cause the guard ring to be less effective to control the potential drop as space charge sign inversion (SCSI) occurs.

Each simulation was performed to $500 \mathrm{~V}$ and no breakdown occured. However, not all details have been included and other processes not included in the simulation could trigger a breakdown at lower bias potential. The highest electrical field are present at the edge of the high voltage electrode and at the surface of the inter-pixel region. 


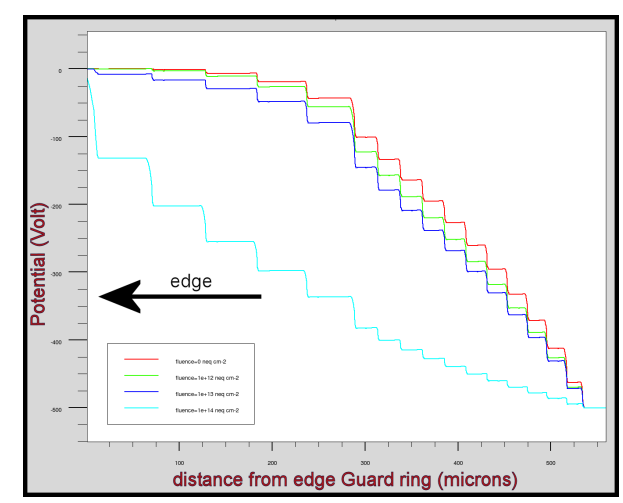

Figure 4. Potential distribution of the guard ring structure for different fluences, unirradiated (red), $1 e 12 n_{\mathrm{eq}} \mathrm{cm}^{-2}$ (green), $1 e 13 n_{\mathrm{eq}} \mathrm{cm}^{-2}$ (dark blue), $1 e 14 n_{\mathrm{eq}} \mathrm{cm}^{-2}$ (pale blue).

\subsection{Dead edge reduction}

Simulation have been performed to explore the possibility to reduce the dead area of the detector that span from the edge to the first guard ring. Simulation for an edge of 100, 200, 300, 465 microns (Actual design) have been performed. Lateral non-depletion width obtained at half-height for these simulation are respectively $0,87,193,330$ microns. The lateral non-depletion width is linear with edge width down to 100 microns where it reaches zero. This suggest that at least 100 microns, plus a security factor, must be used as a dead edge width to prevent lateral breakdown from occuring.

\section{3 reducing the number of guard rings}

The guard ring structure represents inactive area of the detector and must be reduced, The actual ATLAS detector has been simulated with $0,1,3,4$ of the outer guard ring removed.

Figure 6 shows the potential distribution taken by the guard rings for the different simulated structures with an applied bias of $500 \mathrm{~V}$. It is shown that the potential of the guard rings are almost the same as befored their removal, with the outer guard rings moving closer to ground but never reaching it.

\section{Discussion}

Simulation performed in this work show that for unirradiated detector, the lateral depletion of the current ATLAS pixel sensor design biased at $500 \mathrm{~V}$ is sufficient to protect the edge of the detector from lateral breakdown. The choice of a large edge width was conservative choice made to keep a safety margin. Simulation shown in section 5.2 shows that the edge width could be reduced to more than 100 microns while keeping an undepleted zone at the edge of the detector that is sufficiently small, which ensure no edge lateral breakdown. New laser cutting or plasma etching technologies could be used to reduce the thickness or the damaged area at the edge of the detector, making it possible to reduce safely the edge of the detector to a smaller value of the order of 100 microns.

The other approach to reduce the inactive area would be to reduce the number of guard rings in the multi-guard ring structure. The problem that could arise from this approach would be an augmentation of the electric field profile at the guard ring surface. If potential drop between guard 


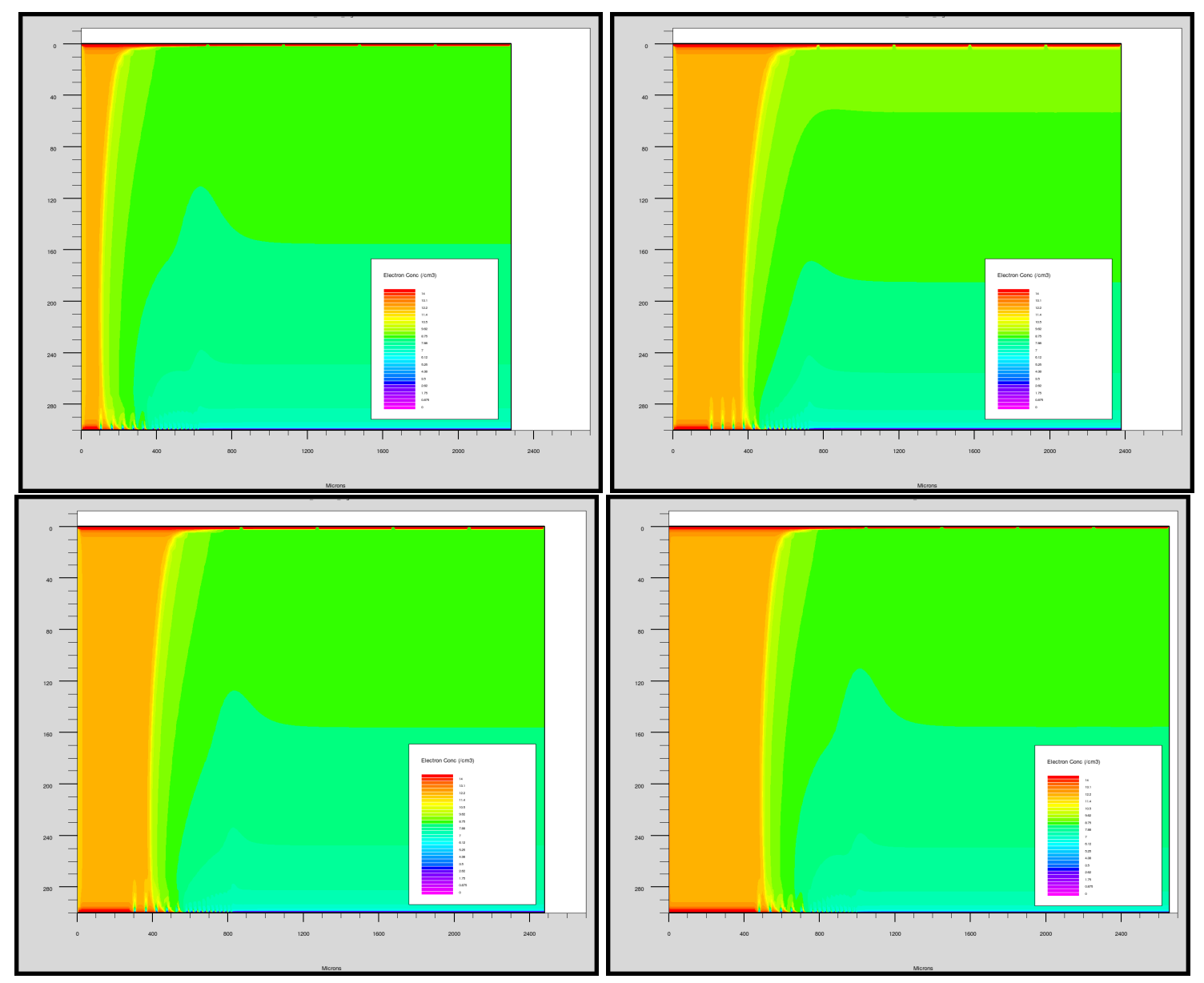

Figure 5. Simulated electron concentration profile for ATLAS pixel detector for a dead edge width of (from left to right, top to bottom): 100, 200, 300 and 468 microns, minimum=0 maximum $=1 \mathrm{e} 14 \mathrm{~cm}^{-3}$.

rings or between a guard ring and the edge become to elevated, the electric field might reach breakdown value for silicon. In the simulation we performed, we show how removing up to 4 of the outer guard rings would affect only slightly the electric field distribution under the guard ring. Also, removing a guard ring do not affect much the potential taken by the other guard ring present in the structure. As we can see in figure 4, the highest potential drop occurs at the fifth outer guard ring. Hence, removing this one would bring a very large potential drop of more than $100 \mathrm{~V}$ between the outer guard ring and the edge of the detector. This would be unacceptable as this would add a possibility of breakdown to the detector.

One concern for n-in-n sensor like actual ATLAS pixel design is the behavior of the guard rings after space charge sign inversion (SCSI). Figure 4 show how potential distribution change as a function of radiation damage. After SCSI, the outer guard ring become more effective to lower the potential toward the edge while inner guard ring become innefective. Removing the outer guard rings would allow the inner guard ring, closer to each other, to become effective and would limit the number of ineffective guard ring after type inversion.

Finally, during this work, it has been noticed that the guard ring behavior is closely linked to 


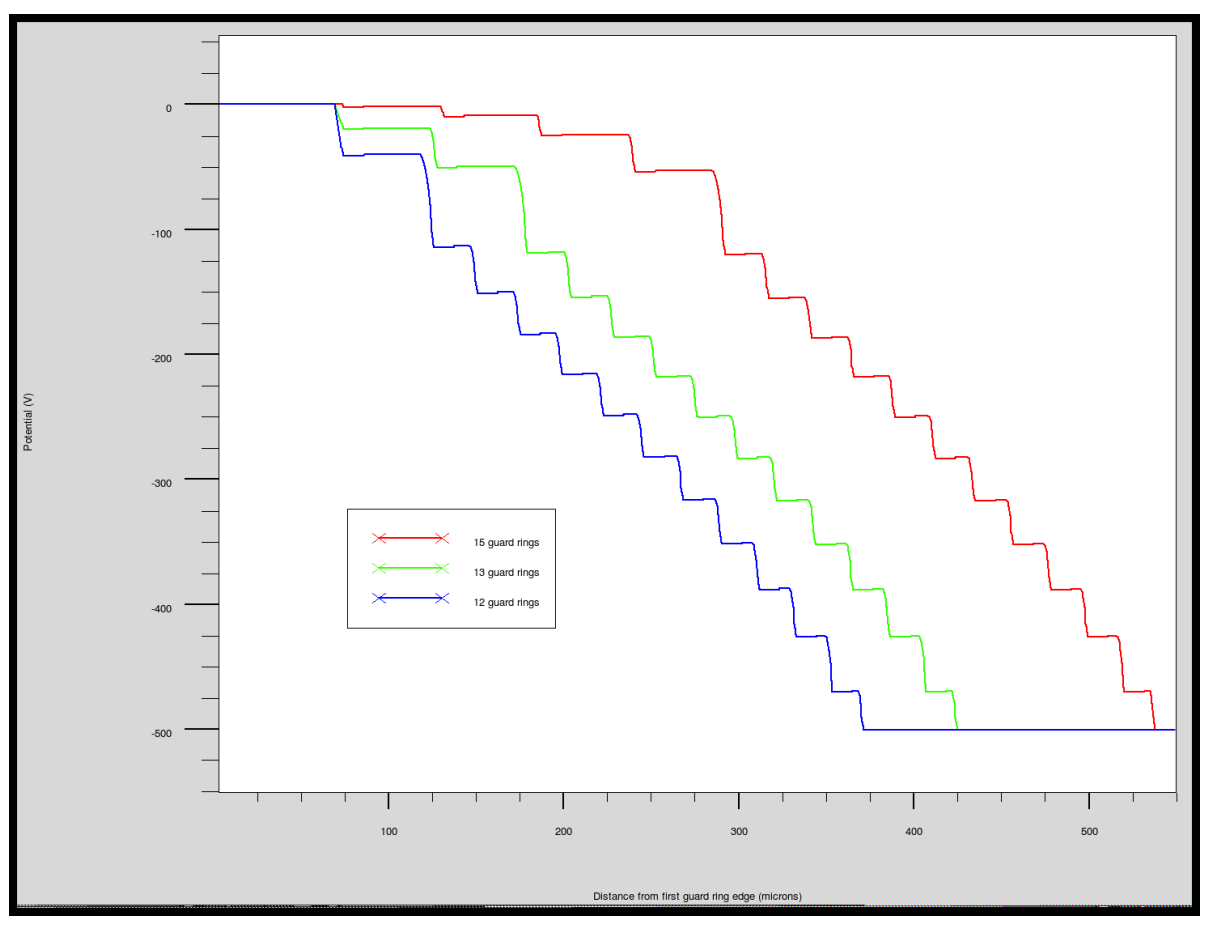

Figure 6. Simulated Potential distribution for actual ATLAS detector with $0,1,3,4$ of the outer guard rings removed.

the doping profiles and oxide charge values that are used for simulation. To be able to perform accurate simulation of the behavior of real device, we need to be able to compare simulated result to measurement in order to fine tune the model's parameters.

\section{Conclusion}

In this work, we presented the work done to understand the physics involved in semiconductor pixel sensor simulation and to assemble a framework for simulation of the actual ATLAS pixel sensor including possible modification to its design. We performed a serie of simulation with modified guard ring structure, edge width and depth, for different amount of exposition to radiation. From the results of the simulation, we obtained two recomendation on the design: Reducing the dead edge to 100 microns and removing up to 4 of the outer guard rings. Test structures will be needed to tune our simulation and validate our results.

\section{References}

[1] ATLAS collaboration, M.S. Alam et al., ATLAS pixel detector: Technical design report, CERN-LHCC-98-013 (1998).

[2] Yu Y. Peter and M. Cardona, Fundamentals of Semiconductors, 3rd Edition, Springer (2003).

[3] F. Moscatelli et al., An enhanced approach to numerical modeling of heavily irradiated silicon devices, Nucl. Instrum. Meth. B 186 (2002) 171. 
[4] Z. Li and H. Kraner, Fast neutron radiation damage effects on high resistivity silicon junction detectors, J. Electron. Mater. 21 (1992) 701.

[5] V. Eremin, Z. Li and I. Ilyashenko, Trapping induced $N_{\text {eff }}$ and electrical field transformation at different temperatures in neutron irradiated high resistivity silicon detectors, Nucl. Instrum. Meth. A 360 (1995) 458.

[6] D. Menichelli, M. Bruzzi, Z. Li and V. Eremin, Modelling of observed double-junction effect, Nucl. Instrum. Meth. A 426 (1999) 135.

[7] S. Selberherr, Analysis and simulation of semiconductor devices, Wien (1984).

[8] M. Moll, Radiation Damage in Silicon Particle Detectors, Ph.D. thesis, University of Hamburg (1999).

[9] Z. Li, W. Huang and L.J. Zhao, Study of the correlation between the cutting edge current breakdownand the simulated lateral electrical field boundary in high resistivitysilicon detectors with multi-guard ring structure, IEEE Trans. Nucl. Sci. 47 (2000) 729.

[10] E. Noschis, V. Eremin and G. Ruggiero, Simulations of planar edgeless silicon detectors with a current terminating structure, Nucl. Instrum. Meth. A574 (2007) 420. 